\title{
De Haas-van Alphen effect in the organic superconductor $\kappa$-(BEDT-TTF $)_{2} I_{3}$
}

\author{
M. Heinecke ${ }^{1}$, K. Winzer ${ }^{1}$, D. Schweitzer ${ }^{2}$ \\ ${ }^{1}$ 1. Physikalisches Institut der Universität Göttingen, Bunsenstrasse 9, D-37073 Göttingen, Germany \\ 2 3. Physikalisches Institut der Universität Stuttgart, Pfaffenwaldring 57, D-70569 Stuttgart, Germany \\ Received: 18 May 1993
}

\begin{abstract}
In the two-dimensional organic superconductor $\kappa$-(BEDT-TTF $)_{2} \mathrm{I}_{3}$ de Haas-van Alphen oscillations were observed at magnetic fields above $5 \mathrm{~T}$ and temperatures between 0.4 and $2 \mathrm{~K}$. We found two dHvA frequencies at $3.846 \mathrm{kT}$ and $0.570 \mathrm{kT}$, which correspond to the cross-sectional areas of the Fermi surface expected from a tight-binding calculation. From the temperature dependence of the oscillation amplitudes the effective mass belonging to the larger orbit was found to be $3.80 \mathrm{~m}_{\mathrm{o}}$. Precise measurements of the angular dependence of the dHvA frequency show no deviation from that expected for a cylindrical Fermi surface. The angular dependence of the amplitude including spin splitting zeroes can essentially be described by a two-dimensional Fermi surface. Certain systematical deviations, however, hint for a slight corrugation.
\end{abstract}

PACS: $71.25 . \mathrm{H} ;$ 74.70.K

\section{Introduction}

Charge-transfer salts based on the electron-donor molecule BEDT-TTF, bisethylendithia - tetrathiafulvalene (abbreviated ET) form a large number of organic superconductors. Salts of the type $(\mathrm{ET})_{2} X$, where $X$ stands for a monovalent anion, are characterized by their layered structure. Different ways of stacking of the ET molecules lead to many polymorphic phases, denoted by $\alpha, \beta, \kappa, \Theta$ etc. The $\kappa$-phase of $(\mathrm{ET})_{2} I_{3}$ has a monoclinic crystal structure with $a=1.639 \mathrm{~nm}, b=0.847 \mathrm{~nm}$, $c=1.283 \mathrm{~nm}$ and $\beta=108.56^{\circ}[1,2]$. The ET molecules are arranged in sheets being parallel to the $b-c$ plane, which are separated from each other by quasi isolating $I_{3}$ sheets. Since electrical conductivity is restricted to the ET sheets, the electronic structure should not depend on wave vector components in $a^{*}$-direction. Tight-binding calculations for the $b^{*}-c^{*}$ plane of the reciprocal lattice show the existence of two bands crossing the Fermi level $[3,4]$, one of these corresponding to an open, the other to a closed semiclassical orbit on the Fermi surface (FS), similar to the situation in other $\kappa$ - or $\Theta$ phase ET salts.

Important informations on the electronic band structure and the FS can be obtained from the observation of magnetic quantum oscillations in Shubnikov-de Haas $(\mathrm{SdH})$ or de Haas-van Alphen (dHvA) experiments. Due to the fact that most of the ET salts crystallize in the form of thin platelets, prevailing $\mathrm{SdH}$ measurements were published [5]. Only a few number of dHvA measurements on high-purity $(\mathrm{ET})_{2} \mathrm{X}$ crystals [6-8] were published up to now.

The $\kappa$-phase of $(\mathrm{ET})_{2} \mathrm{X}$ is of special interest, because organic superconductors with the highest $T_{C}$ know belong to this group (for $X=\mathrm{Cu}\left[\mathrm{N}(\mathrm{CN})_{2}\right] \mathrm{Cl}, T_{C}=12.5 \mathrm{~K}$ at $0.3 \mathrm{kbar}$ ). For some of these substances, dHvA or $\mathrm{SdH}$ effect measurements have confirmed the picture of a cylindrical FS $[4,6]$.

In the triclinic $\beta$-phase of the ET salts, however, anisotropy effects in the magnetoresistance and in the fundamental frequency of quantum oscillations occurred and were explained assuming that the FS is slightly modulated in the direction perpendicular to the conducting layers [9-11].

In this paper we present the first $\mathrm{dHvA}$ measurements on $\kappa$-(ET) ${ }_{2} I_{3}$ with a detailed analysis of the angular dependence of the dHvA amplitudes, showing that also in the $\kappa$-phase a small corrugation of the FS is observable.

\section{Experiment}

High-purity single crystals of $k$-(ET) $I_{2} I_{3}$ were grown by electrochemical oxidation of ET in 112-TCE resulting in plate-shaped samples of up to $2 \mathrm{~mm}$ side length and smaller thickness [12]. For a number of samples the superconducting transition was measured inductively. The samples with the highest values of $T_{C} \approx 3.6 \mathrm{~K}$ and the smallest transition widths of $\Delta T_{\mathrm{C}} \approx 0.2 \mathrm{~K}$ were selected for the dHvA-measurements. The directions of the 
crystal axes were determined from the angles between neighbouring crystal faces. The mounted crystals could be orientated with an accuracy of about $1^{\circ}$.

The dHvA effect was measured in a ${ }^{3} \mathrm{He}$ bath cryostat, which is equipped with a superconducting $\mathrm{NiTi} / \mathrm{Nb}_{3} \mathrm{Sn}$ coil. It allows measurements in magnetic flux densities up to $13 \mathrm{~T}$ at temperatures between 0.35 and $3.2 \mathrm{~K}$ [13]. The dHvA signal was detected as a voltage induced on a pair of compensated pick-up coils by field- or temperature modulation, respectively [14].

For measurements using the field modulation technique, a modulation field $b_{0}(f=158 \mathrm{~Hz})$ of about $100 \mathrm{mT}$ was applied parallel to the slowly varying field $B$. The dHvA signal was detected at $2 f$ with a lock-in amplifier and registered and stored in equidistant steps of $1 / B$. Two different sample holders were used in which a defined sample rotation was possible during the experiment with a rotation axis perpendicular to $B$. They were used for measurements at fixed sample orientation in the slowly varying field $B$ and for measurements with a rotating sample at fixed magnetic field, respectively.

For temperature modulation the samples were illuminated with a chopped beam of a laser diode, which was dispensed through a flexible light guide. A chopper frequency $f \approx 1 \mathrm{kHz}$ was used to obtain a good signal-tonoise ratio. The dHvA signal was detected at the modulation frequency $f$. To ensure a stable optical coupling, the samples were fixed rigidly inside the detection coils and could not be rotated in situ. Using this technique, measurements were made with $B \| a^{*}$.

\section{Results and discussion}

Three different samples of $\kappa$-(ET) $I_{3} I_{3}$ were investigated, which showed dHvA oscillations of comparable amplitude. Figure 1 shows the dHvA signal vs. magnetic field $B$, measured using temperature modulation at a mean temperature $\langle T\rangle=0.50 \mathrm{~K}$ in the field range $7.5 \ldots 12 \mathrm{~T}$ with $B \| a^{*}$. Obviously there are two fundamental fre-

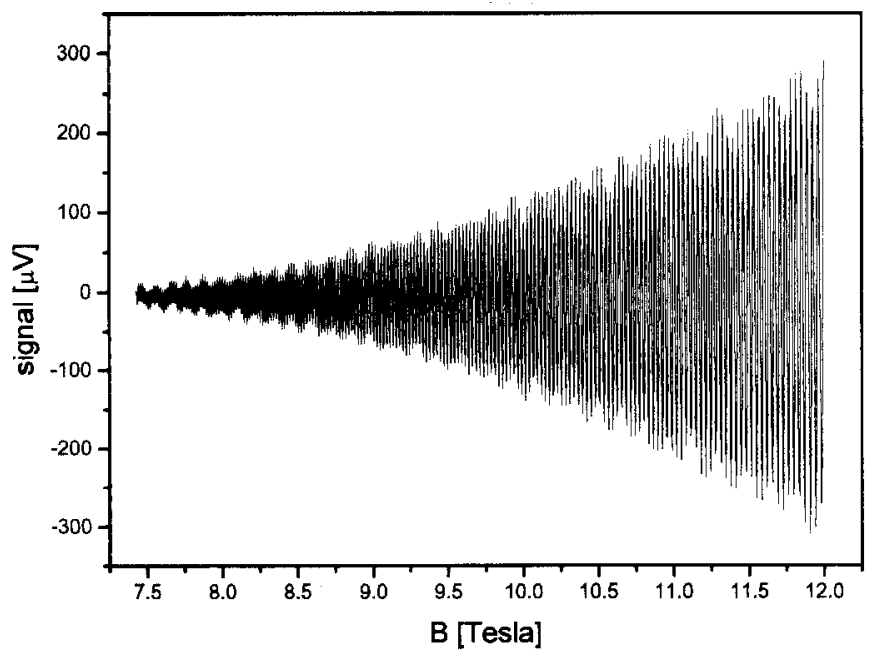

Fig. 1. dHvA signal of $\kappa$-(ET) $I_{2} I_{3}$ vs. magnetic field $B$, measured using temperature modulation at a mean temperature $\langle T\rangle=0.50 \mathrm{~K}$ with $B \| a^{*}$ quencies: a lower one with a nearly constant amplitude and a higher one, with a strongly growing amplitude with increasing field. Both oscillation modes could be detected for magnetic fields down to $5 \mathrm{~T}$.

The fundamental frequencies were calculated to be $F_{1}=0.57 \mathrm{kT}$ and $F_{2}=3.85 \mathrm{kT}$ by a Fourier transform algorithm as shown in Fig. 2. The corresponding extremal cross section areas $A=(2 \pi e / \hbar) F$ of the FS are $A_{1}$ $=5.4 \mathrm{~nm}^{-2}$ and $A_{2}=36.7 \mathrm{~nm}^{-2}$, respectively. A more precise value of the frequency $F_{2}=3.8460 \mathrm{kT}$ was obtained by linear regression from a plot of the number of Landau levels vs. the reciprocal field as shown in Fig. 3.

The electronic structure of $\kappa$-(ET) $I_{3}$ is determined by the overlap of the electronic wave functions between the ET molecules in the sheets parallel to the $b-c$ plane. The strength of the side-by-side interaction becomes comparable to that of the face-to-face interaction between the ET molecules, resulting in a purely two-dimen-

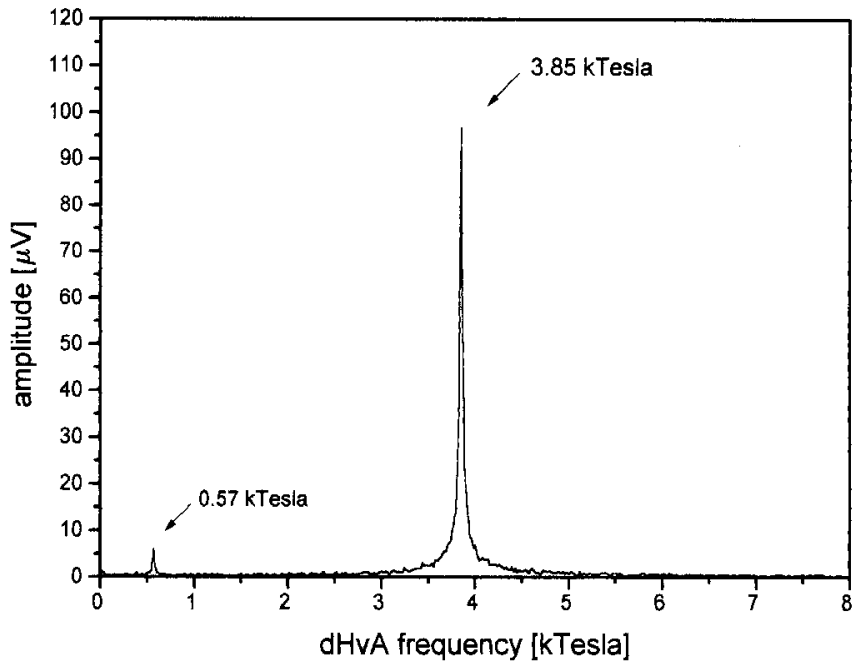

Fig. 2. Fast Fourier transform of the data from Fig. 1 between 7.5 and $12 \mathrm{~T}$

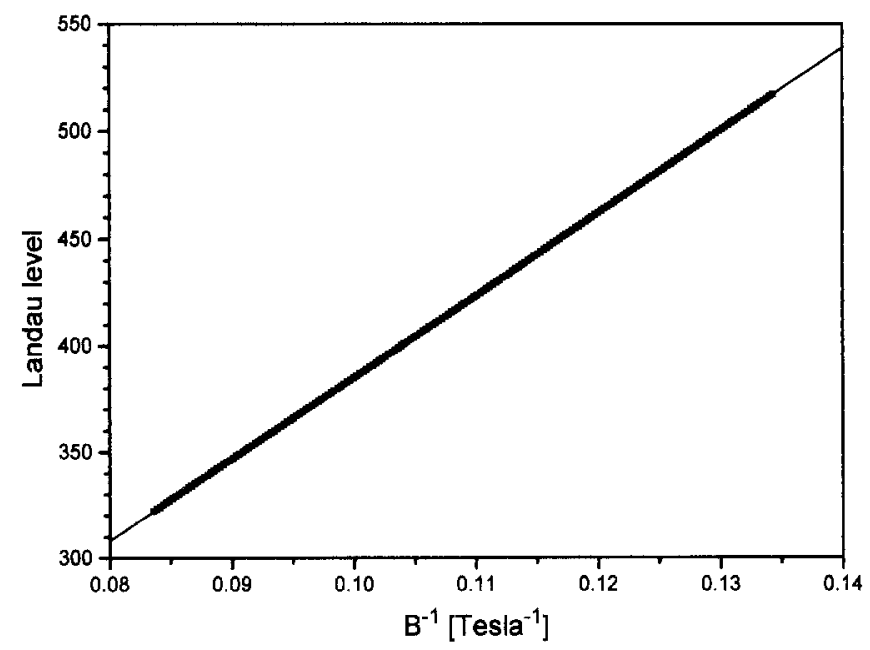

Fig. 3. Number of Landau levels vs. reciprocal field $1 / B$ for $B \| a^{*}$. The solid line is a linear fit yielding $F_{2}(0)=3.8460 \mathrm{kT}$ 
sional conducting sheet. There are four ET molecules per unit cell. Considering that one electron per two ET molecules is transferred to one $I_{3}$ molecule, the density of holes is determined to be $n=2 / V_{u c}$. In a simple free electron picture we expect a cylindrical FS with $k_{F}$ $=2 \pi \sqrt{n / a^{*}}=3.4 \mathrm{~nm}^{-1}$. The cross section of the FS $A$ $=36.3 \mathrm{~nm}^{-2}$ corresponds to the cross section of the first Brillouin zone $A_{B Z}=b^{*} c^{*}$ and agrees with the measured cross section $A_{2}=36.7 \mathrm{~nm}^{-2}$ within one percent. In the $c^{*}$ direction, as $k_{F}>c^{*}$, the closed cylindrical FS intersects the zone boundary, which should lead to a small bandgap. The expected FS therefore contains a closed hole-like cylindrical surface with an elliptical cross section localized around $Z$ and an electron-like open surface along $c^{*}$.

In the simple free electron picture, the cross section of the elliptical orbit around $Z$ can be approximated by two circle segments $A_{Z}=(\alpha-\sin \alpha) k_{F}^{2}$ with $\cos (\alpha / 2)$ $=c^{*} / 2 k_{F}$. The calculated cross section $A_{Z}=6.21 \mathrm{~nm}^{-2}$ is only about $10 \%$ larger than the measured cross section $A_{1}=5.4 \mathrm{~nm}^{-2}$.

Kajita et al., Oshima et al. $[3,4]$ have calculated the band structure and the FS of $\kappa$-(ET) $I_{3}$ as shown in Fig. 4 using a tight-binding method based on the extended Hückel approximation. As $\kappa-(\mathrm{ET})_{2} I_{3}$ belongs to the $P 2_{1} / c$ space group, the two conduction bands found are degenerated along the $Z-M$ zone boundary, leading to an elliptical hole orbit around $Z$ and a nearly ciruclar hole orbit around $\Gamma$, which crosses the zone boundary.

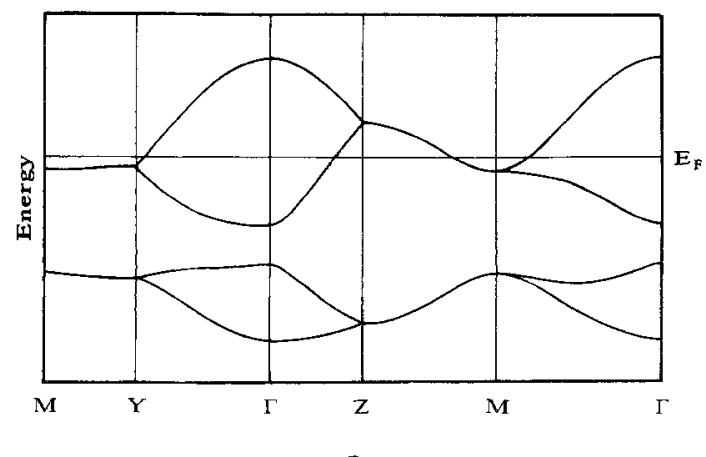

a
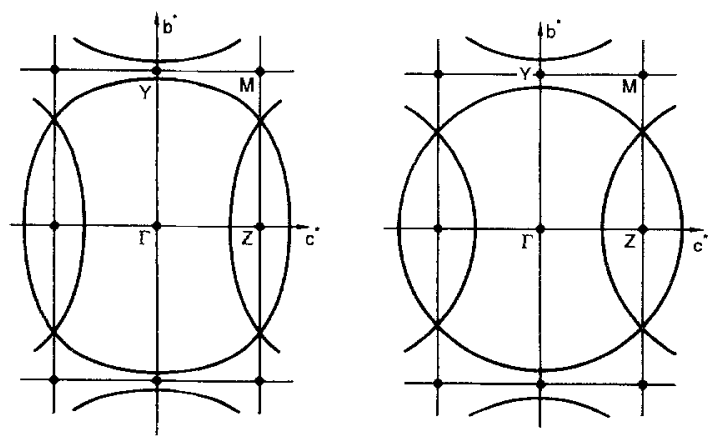

b C

Fig. 4a-c. Energy band structure a and cross section of the FS b of $\kappa$-(ET) ${ }_{2} I_{3}$ from Kajita et al. [4]. For comparison the FS in a simple free electron picture is also shown $\mathbf{c}$
Comparing the measured data with the cross sections resulting from the band structure calculations $[3,4]$ for the $b^{*}-c^{*}$ plane, one finds good agreement. The smaller frequency $F_{1}$ can be related to the small hole pocket belonging to the upper conduction band at the $Z$ point. $F_{2}$ belongs to the orbit around $\Gamma$.

In other $\kappa$-type salts like $\kappa$-(ET) ${ }_{2} \mathrm{Cu}(\mathrm{NCS})_{2}$, the degeneracy mentioned above is removed due to a lack of a center of symmetry, and the orbit around $\Gamma$ occurs only as a magnetic breakdown effect at high fields [15]. So, if there should be an energy gap $E_{\mathrm{g}}$ also in $\kappa$-(ET) ${ }_{2} I_{3}$, we can estimate an upper limit using the Blount criterion [16] $B>m^{*} E_{g}^{2} / e \hbar E_{F}$. As $F_{2}$ oscillations were observed down to $5 \mathrm{~T}, E_{\mathrm{g}}$ must be smaller than about $5 \mathrm{meV}$.

In the measurements which were performed in the temperature modulation technique, both frequencies $F_{1}$ and $F_{2}$ could be observed, while in the experiments with field modulation preferentially the higher frequency $F_{2}$ occurred. This can be understood considering that in the field modulation technique the $\mathrm{dHvA}$ amplitudes are proportional to the Bessel function $J_{2}(x)$ with $x$ $=2 \pi F b_{0} / B^{2}[16]$. For $x \ll 1$ the Bessel function can be approximated by $J_{2}(x) \approx x^{2} / 2$ leading to a strong suppression of $\mathrm{dHvA}$ oscillations with small frequencies. In the temperature modulation technique the amplitudes depend linearly on the dHvA frequencies [16]. Hence the temperature modulation technique is more suitable for detecting small dHvA frequencies.

In the high field region between 10 and $12 \mathrm{~T}$ the dHvA signal is dominated by the higher frequency $F_{2}$ in both cases. Using the field modulation technique, the amplitude of $F_{2}$ was measured for at least eight different temperatures. The temperature dependence of the amplitude was fitted to the temperature damping factor of the Lifshitz-Kosevich formula

$R_{T}=\frac{r z}{\sinh r z} \quad$ with $z=\lambda \frac{m_{c}}{m_{0}} \frac{T}{B} \quad$ and

$\lambda=14.693 \frac{\mathrm{T}}{\mathrm{K}}$.

$r$ is the number of the dHvA harmonic being 1 for the fundamental. From the slope of the corresponding plot (Fig. 5a) the cyclotron mass $m_{c}$ was calculated to be $m_{c}$ $=3.80 \mathrm{~m}_{0}$.

With the value of the cyclotron mass known, the Dingle temperature $T_{D}$ can be obtained from the magnetic field dependence of the oscillation amplitude in Fig. 1. The field dependence of the amplitude $A_{\chi}$ of susceptibility oscillations is given by

$A_{\chi}=$ const. $T B^{-5 / 2} R_{D} / \sinh z$,

where $R_{D}$ is the Dingle damping factor

$R_{D}=\exp \left(-r \lambda \frac{m_{c}}{m_{0}} \frac{T_{D}}{B}\right)$.

The sensitivity factor for the temperature modulation signal [16] 

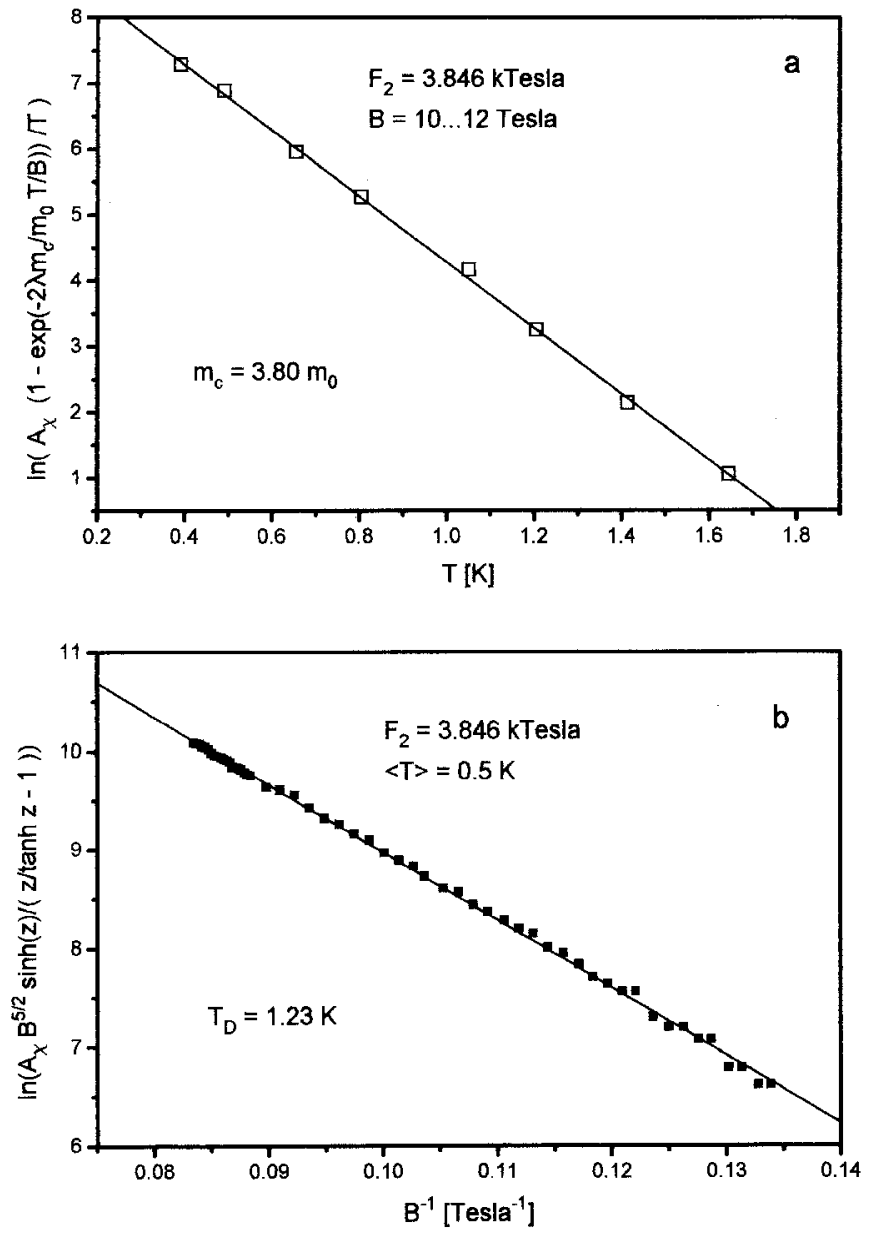

Fig. 5. a Temperature dependence of the dHvA amplitude of the frequency $F_{2}=3.85 \mathrm{kT}$. b dHvA amplitude of Fig. 1 vs. inverse magnetic field $1 / B$. The Dingle temperature was obtained from the slope of the straight line

$f_{T}=\frac{z}{\tanh z}-1$

depends also on the magnetic field and has to be included. From a corresponding plot of $\ln \left(A_{\chi} B^{5 / 2} \sinh z /(z / \tanh z-1)\right)$ vs. $1 / B$, which is given in Fig. $5 \mathrm{~b}$, a Dingle temperature of $T_{D}=1.23 \mathrm{~K}$ was obtained. It should be noted that the data can be fitted nearly perfectly by a straight line in the whole range $7.5 \mathrm{~T}<B<12 \mathrm{~T}$ showing no indication for magnetic breakdown effects.

To study the geometry of the FS in more detail, field modulation measurements were made for $B \perp b$ and $B \perp c$. For fixed angles of inclination $\varphi$ between $B$ and the $a^{*}$ axis, the dHvA signal was detected in the field range $10 \mathrm{~T}<B<12 \mathrm{~T}$ at $0.5 \mathrm{~K}$. $F_{2}$ oscillations could be observed at angles up to $60^{\circ}$. Figure 6 shows the measured angular dependence of the dHvA frequency $F_{2}$ for $B \perp c$. The data follow perfectly the behaviour $F_{2}(\varphi)$ $=F_{2}(0) / \cos \varphi$, shown by the solid line, as expected for a cylindrical FS.

The angular dependence of the $\mathrm{dHvA}$ amplitudes for $B \perp c$ which is plotted in Fig. 7 shows a strongly anisotropic behaviour. At certain angles $\left(15.4^{\circ}, 37.3^{\circ}, 48.5^{\circ}\right.$ and $55^{\circ}$ ) only very weak oscillations could be detected. The results were found to be the same for $B \perp b$.

A similar anisotropy occurred observing the angular dependence of the $\mathrm{dHvA}$ oscillations by rotating the sample in a fixed field. Figure 8 shows a corresponding plot at $B=12 \mathrm{~T}, T=0.5 \mathrm{~K}$ and the rotation axis in the $b-c$ plane. The envelope of the dHvA oscillations has a similar shape as the amplitudes in Fig. 7. Especially its minima occur at the same angles.

The vanishing of the signal or of its envelope, respectively, is caused by the spin splitting of the Landau levels leading to two sets, which are separated by the energy $\Delta \varepsilon=(g / 2)\left(e h / m_{0}\right) B$, where $g$ is the $g$-factor averaged over a cyclotron orbit. The Landau levels themselves are separated by $\hbar \omega_{c}$. Therefore, the measured magnetization is the superposition of two sets of oscillations shifted by $\Phi=2 \pi \Delta \varepsilon / \hbar \omega_{c}$ yielding the spin splitting factor

$R_{S}=\cos \left(\frac{\pi}{2} r g \mu_{b}\right)$,

of the Lifshitz-Kosevich formula, where $\mu_{b}=m_{b} / m_{o}$ is the reduced band structure effective mass. The band mass is given by $m_{b}=\left(\hbar^{2} / 2 \pi\right)(\partial A / \partial E)$. Therefore, the angular dependence of the extremal area $A$ of the FS should also be reflected in

$m_{b}(\varphi)=\frac{m_{b}(\varphi=0)}{\cos \varphi}$

Assuming $g$ to be angular independent and $m_{b}$ to have the form (6), the spin splitting factor $R_{S}$ vanishes for angles $\varphi_{v}$

$\varphi_{\nu}=\arccos \frac{g \mu_{b}(0)}{2 v-1}$,

where $v$ is an integer. Taking the average of the corresponding minima in Fig. 7 and those of the envelope in Fig. 8 one finds the angles $15.4^{\circ}, 37.5^{\circ}, 48.5^{\circ}, 54.9^{\circ}$ and $58.1^{\circ}$. From a plot of $1 / \cos \varphi$ vs. $(2 v-1)$ we obtain

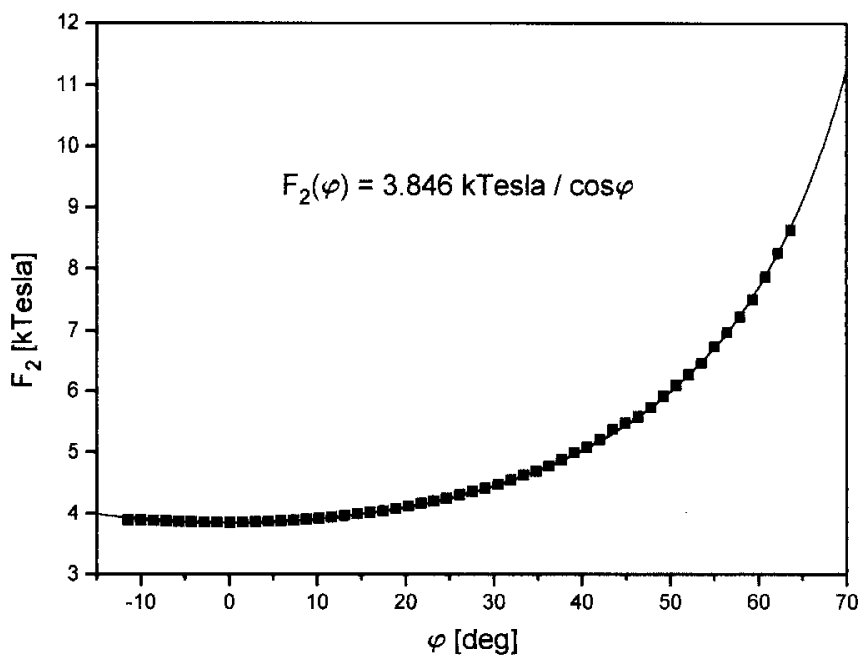

Fig. 6. Angular dependence of the dHvA frequency $F_{2}$ for $B \perp_{c}$. Solid line: calculated curve $F_{2}(\varphi)=F_{2}(0) / \cos \varphi$ 


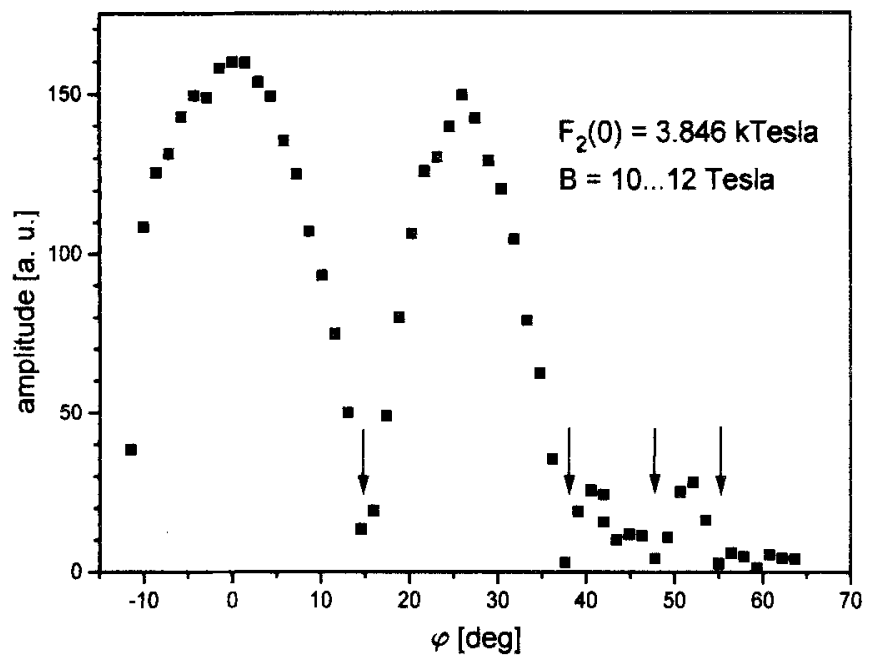

Fig. 7. Angular dependence of the $F_{2}$ amplitude for $B \perp c$. The arrows indicate the positions of the spin splitting zeroes

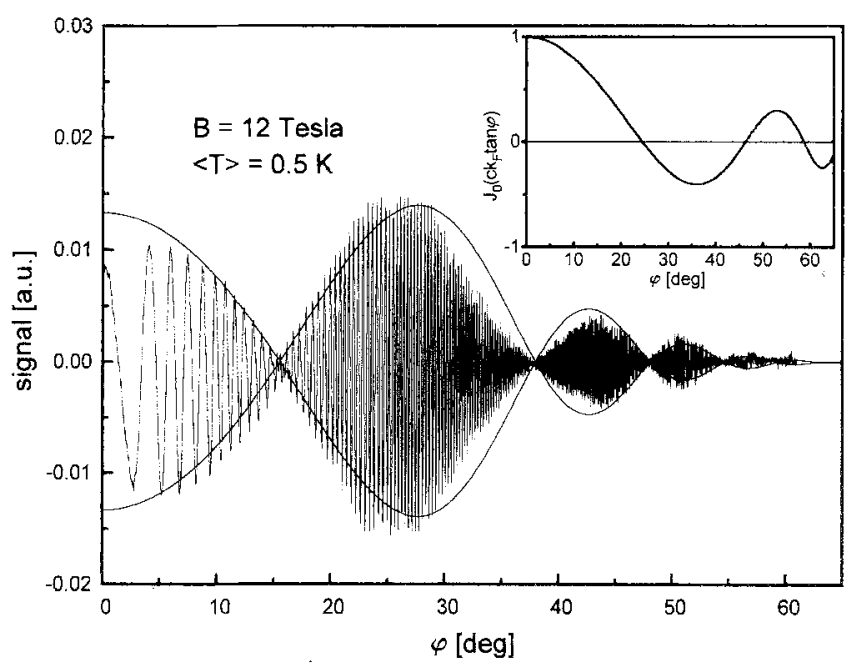

Fig. 8. Angular dependence of the dHvA signal obtained by rotating the sample in a fixed field of $12 \mathrm{~T}$. The envelope was calculated from The Lifshitz-Kosevich formula with $m(\varphi)=m(0) / \cos \varphi ; T_{D}$ $=0.49 \mathrm{~K}$ and $g \mu_{0}(0)=8.63$.

Inset: Angular dependence of the Bessel function $J_{0}\left(c k_{F} \tan \varphi\right)$ with $c=1.55 \mathrm{~nm}$ and $k_{F}=3.40 \mathrm{~nm}^{-1}$

$g \cdot \mu_{b}(0)=8.63$. With the measured effective mass $m_{b}(0)$ $=3.80 \mathrm{mo}_{o}$ a $g$-factor of $g=2.27$ results. This value is considerably larger than the $g$-factor measured by spin resonance. Angular dependent measurements on $\alpha$ - and $\beta$-(ET) $I_{3} I_{3}$ yield values of $g_{S}=2.004 \ldots 2.011$ [17]. The $g$ factor in the Lifshitz-Kosevich formula is renormalized by many-body effects like electron-electron (EE) or electron-phonon (EP) interactions, while $g_{S}$ includes only spin orbit coupling but no many-body effects. For the $g$-factor Kaplan et al. [18] have shown that the renormalization is simply $g=g_{S} /\left(1+B_{0}\right)$. The coefficient $B_{0}$, which is determined by both EE and EP effects, is typically small and negative. We find $B_{0}=-0.12$ for $\kappa$-(ET) $I_{3}$, comparable to values obtained for noble metals.
The details of the rotation diagram given in Fig. 8 are in good agreement with the predictions of the Lifshitz-Kosevich formula: In the region of the first minimum at about $15^{\circ}$ a large harmonic content of the amplitudes is observed. Obviously, at this angle the spin splitting factor (5) is close to zero for the fundamental $(r=1)$ and, consequently, maximal for the second harmonic $(r=2)$. The same argument is valid for the second and higher minima, but due to the increasing effective mass and its influence in the other damping factors (1) and (3), the second harmonic content is strongly reduced for larger angles.

If a weak interlayer interaction between adjacent conducting layers is present, the cylindrical FS should be slightly modulated. In the simplest way, such a warped FS can be described by

$\varepsilon_{k}=\frac{\hbar^{2}}{2 m}\left(k_{x}^{2}+k_{y}^{2}\right)-2 t \cos \left(c k_{z}\right)$

where $k_{x}$ and $k_{y}$ are the components of the wave vector $k$ in the conducting plane, $t$ is the interlayer transfer energy and $c$ is the spacing between adjacent layers. Yamaji [10] has shown that the angular dependence of the frequency corresponding to such a warped FS can be expressed as

$F(\varphi) \cos \varphi=F(0)+\Delta F(0) \cdot J_{0}\left(c k_{F} \tan \varphi\right)$,

where $J_{0}$ is the Bessel function of zeroth order and

$\Delta F(0)=\frac{2 m t}{e \hbar} \cos \left(c k_{z}\right)$

depends on the transfer energy $t$. The second term in Eq. (9) vanishes periodically for the values of $\varphi$ satisfying

$c k_{F} \tan \varphi=\pi\left(n-\frac{1}{4}\right)$

with integer $n$. For these values of $\varphi$ all orbits on the FS have the same orbital areas. This leads to a larger degeneracy of the Landau levels compared to the usual $3 D$ case. Kajita et al. [19] have shown that at these angles the magnetoresistance of $\Theta-(\mathrm{ET})_{2} I_{3}$ reveals local minima periodically in $\tan \varphi$. The same effect was reported for $\beta$-(ET) ${ }_{2} \mathrm{IBr}_{2}$ by Kartsovnik et al. [9]. Furthermore, they found strong $\mathrm{SdH}$ oscillations with maximum amplitudes at the above mentioned angels.

A modulated FS leads to the occurrence of more than one frequency and of a distinctive angular behaviour of the frequency difference. Such a behaviour with at least two frequencies and nodes with nearly zero remaining amplitudes as a function of the magnetic field was recently reported on $\beta$-(ET) ${ }_{2} \mathrm{IBr}_{2}[20]$.

In $\kappa-(E T)_{2} I_{3}$ the effect of a corrugated FS seems to be very small. There are no indications of nodes in the field dependence of the amplitude. From Fig. 8 we could obtain a precise determination of the angular dependence of the dHvA frequency: One oscillation of the dHvA signal corresponds to a frequency difference of $\Delta F=B$ or to a relative change of $\Delta F / F(0)=3.120 \cdot 10^{-3}$. By counting the number of oscillations up to angles of 
$45^{\circ}$ we obtain $F(\varphi) \cdot \cos \varphi=F(0)\left(1 \pm 10^{-3}\right)$. This result shows that the FS of $\kappa$-(ET) $I_{2} I_{3}$ has a nearly perfect cylindrical form with a negligible amount of warping.

However, the angular dependence of the dHvA amplitudes in Fig. 8 gives a hint to a very weak corrugation of the FS. The envelope of the oscillations can be calculated from the product of the three damping factors $R_{T}$ (Eq.(1)), $R_{D}$ (Eq.(3)) and $R_{S}$ (Eq. (5)) as a function of the angle $\varphi$. Using the angular dependence of $m_{b}(\varphi)$ from (6) and regarding $g \mu_{b}$ and $T_{D}$ as fitting parameters to reproduce the angles of the spin zeroes and the total angular dependence of the amplitudes, respectively, we obtain quite a good agreement. A closer comparison between the measured amplitudes and the calculated envelope shows that around $\varphi \approx 25^{\circ}$ the amplitudes are larger, whereas they are smaller than the envelope around $\varphi \approx 0^{\circ}$ and $\varphi \approx 35^{\circ}$. This behaviour can be attributed to the angular dependence of the Bessel function $J_{0}\left(c k_{F} \tan \varphi\right)$ in Eq.(9). In $\kappa$-(ET) ${ }_{2} I_{3}$ the spacing $c$ between adjacent layers is given by $a \cdot \cos \beta=1.55 \mathrm{~nm}$ and the Fermi wave vector calculated within the free electron picture is $k_{F}=3.40 \mathrm{~nm}^{-1}$. The angular dependence of the Bessel function $J_{0}(5.28 \cdot \tan \varphi)$ is shown in the inset of Fig. 8. It can be seen that the measured signal exceeds the envelope near the zero values of $J_{0}\left(24.6^{\circ}\right.$ and $\left.46.4^{\circ}\right)$, while it is smaller where $J_{0}$ reaches an extremum $\left(0^{\circ}\right.$ and $36^{\circ}$ ). Due to the small corrugation of the FS the semiclassical electron orbits have a small but finite width of distribution in their areas. At those angles which correspond to the zero values of $J_{0}$, the width of the area distribution function vanishes leading to a complete discretization into Landau levels and hence an increase of the dHvA amplitude.

The above results show that even in the case of a nearly perfect cylindrical FS without a measurable dispersion of the dHvA frequency, an extremely small corrugation can effect the more sensitive amplitudes.

\section{Summary}

In conclusion, the organic superconductor $\kappa$-(ET) ${ }_{2} I_{3}$ shows the characteristic $1 / \cos \varphi$ behaviour of the extremal areas of the FS as expected for layered structure. The measured cross sections of the FS in the $b-c$ plane are in good agreement with $2 D$ band structure calculations. The angular dependence of the dHvA amplitude is dominated by spin-splitting effects of the Landau lev- els. Certain systematical deviations of the dHvA signal from the calculated angular dependence of the amplitude, however, give a clear indication of an extremely small corrugation of the FS.

The authors are very grateful to H.W. Helberg for his help in the sample orientation and N. Thier-Weigang for the development of the temperature modulation technique. The support of the Deutsche Forschungsgemeinschaft is gratefully acknowledged.

\section{References}

1. Saito, G., Enoki, T., Toriumi, K., Inokuchi, H.: Solid State Commun. 42, 557 (1982)

2. Kobayashi, A., Kato, R., Kobayashi, H., Moriyama, S., Nishino, Y., Kajita, K., Sasaki, W.: Chem. Lett. 1987, 459 (1987)

3. Kajita, K., Nishio, Y., Moriyama, S., Sasaki, W., Kato, R., Kobayashi, H., Kobayashi, A.: Solid State Commun. 64, 1279 (1987)

4. Oshima, K., Mori, T., Inokuchi, H., Urayama, H., Yamochi, H., Saito, G.: Phys. Rev. B38, 938 (1988)

5. Tokumoto, M., Swanson, A.G., Brooks, J.S., Agosta, C.C., Hannahs, S.T., Kinoshita, N., Azai, H., Tamura, M., Tajima, H., Kuroda, H., Anderson, J.R.: In: Organic superconductivity, p. 167 Kresin, V.Z., Little, W.A. (eds.) New York: Plenum Press 1990

6. Tokumoto, M., Swanson, A.G., Brooks, J.S., Tamura, M., Tajima, H., Kuroda, H.: Solid State Commun. 75, 439 (1990)

7. Wosnitza, J., Crabtree, G.W., Wang, H.H., Carlson, K.D., Vashon, N.D., Williams, J.M.: Phys. Rev. Lett. 67, 263 (1991)

8. Wosnitza, J., Crabtree, G.W., Wang, H.H., Geiser, U., Williams, J.M., Carlson, K.D.: Phys. Rev. B45, 3018 (1992)

9. Kartsovnik, M.V., Kononovich, P.A., Laukhin, V.N., Shchegolev, I.F.: JETP Lett. 48, 541 (1988)

10. Yamaji, K.: J. Phys. Soc. Jpn. 58, 1520 (1989)

11. Kang, W., Montambaux, G., Cooper, J.R., Jérome, D., Batail, P., Lenoir, C.: Phys. Rev. Lett. 62, 2559 (1989)

12. Kahlich, S., Schweitzer, D., Heinen, I., En Lan, S., Nuber, B., Keller, H.J., Winzer, K., Helberg, H.W.: Solid State Commun. 80, 191 (1991)

13. Behler, S., Winzer, K.: Z. Phys. B 82, 355 (1990)

14. Thier-Weigang, N.: Dissertation, Göttingen 1992

15. Sasaki, T., Sato, H., Toyota, N.: Solid State Commun. 76, 507 (1990)

16. Shoenberg, D.: Magnetic Oscillations in Metals. Cambridge: Cambridge University Press 1984

17. Venturini, E.L., Azevedo, L.Z., Schriber, J.E., Williams, J.M., Wang, H.H.: Phys. Rev. B 32, 2819 (1985)

18. Kaplan, J.A., Glasser, M.L.: Phys. Rev. 186, 958 (1969)

19. Kajita, K., Nishino, Y., Takahashi, T., Sasaki, W., Kato, R., Kobayashi, H., Kobayashi, A., Iye, Y.: Solid State Commun. 70, $1189(1989)$

20. Wosnitza, J.: Int. J. Mod. Phys. B (in press) 